\title{
Allergy to Hymenoptera venoms
}

\author{
Hanna Trzcinska', Sebastian Derdowski², Bartosz Kozlowski², Lukasz Sielski³ \\ Grzegorz Przybylski ${ }^{4}$ \\ ${ }^{1}$ Chair and Department of Pedagogy and Nursing Didactics, L. Rydygier Collegium Medicum, Bydgoszcz, Poland; \\ Nicolaus Copernicus University, Torun, Poland \\ 2 Student Science Circle, Public Health Department, Collegium Medicum, Bydgoszcz, Poland; Nicolaus Copernicus \\ University, Torun, Poland \\ ${ }^{3}$ Chair and Clinic of Rehabilitation, L. Rydygier Collegium Medicum, Bydgoszcz, Poland; Nicolaus Copernicus University, \\ Torun, Poland \\ ${ }^{4}$ Department of Respiratory Medicine and Tuberculosis, L. Rydygier Collegium Medicum, Bydgoszcz, Poland; Nicolaus \\ Copernicus University, Torun, Poland
}

Trzcinska H, Derdowski S, Kozlowski B, Sielski L, Przybylski G. Allergy to Hymenoptera venoms. J Pre-Clin Clin Res. 2013; 7(1): 13-18.

\begin{abstract}
I Abstract
Objective: The aim of this study was to analyze current evidence regarding allergy to Hymenoptera venoms.

Brief review: The Hymenoptera species, which are most important in the context of venom allergy, include the common wasp (Vespula vulgaris), German wasp (Vespula germanica), and the honey bee (Apis mellifera). Depending on the population, systemic allergic reactions occur in $1.5-8.9 \%$ of cases. Every year, anaphylactic reactions cause about 40 deaths in the United States and approximately 100 deaths in Europe. Although venoms of various insects differ in terms of their antigen composition, they can show cross-reactivity. Allergic reactions are classified as local or systemic. The lgE-dependent reaction constitutes the most frequent mechanisms responsible for insect venom hypersensitivity. Diagnosis of hypersensitivity is mostly based on history and positive results of skin tests and/or the presence of specific lgE in serum. Intramuscular injection of adrenaline is the procedure of choice in the case of anaphylactic reaction. Immunotherapy has an established the efficacy in the prevention of an anaphylactic reaction in individuals who are sensitive to Hymenoptera venoms. At present, recombinant antigens are being studied in order to improve the safety and effectiveness of diagnosis and therapy. Conclusion: Hymenoptera venom allergy is a subject of extensive research. There is a need to educate society and medical personnel regarding the management of patients who are sensitive to Hymenoptera venom.
\end{abstract}

\section{Key words}

allergy, Hymenoptera venoms

\section{Abbreviations}

CCD-CarbohydrateCross-reacting Determinants;MCD-peptide-mastcell degranulating peptide;RAST-radioallergosorption test

\section{OBJECTIVE}

The aim of the study was to analyze the current evidence regarding allergy to Hymenoptera venoms.

\section{CURRENT EVIDENCE}

Taxonomy. Hymenoptera is an order comprising various families, with Apidae, Vespidae, and Myrmicidae being important in the context of venom hypersensitivity [1]. In practice, the most important species include the common wasp (Vespula vulgaris), German wasp (Vespula germanica), and the honey bee (Apis mellifera). Moreover, members of Polistes spp. are relatively frequent in Mediterranean countries. Bumblebees are relatively less aggressive; however, exposure to these insects which are used for plant pollination, has also been reflected by reports of allergic reactions resulting from stings inflicted by these representatives of Apidae [2]. Fire ants, belonging to Solenopsis spp., are the most important representatives of Myrmicidae, but are not found in Poland [3].

Address for correspondence: Hanna Trzcinska, Chair and Department of Pedagogy and Nursing Didactics, Nicolaus Copernicus University in Torun, Ludwik Rydygier Collegium Medicum in Bydgoszcz, Techników 3, 85-801 Bydgoszcz, Poland e-mail: hanna.trzcinska@cm.umk.pl

Received: 10 December 2012; accepted: 17 June 2013
Prevalence. According to retrospective studies involving adult populations, systemic reaction occurs in $1.5-5.3 \%$ of stung individuals. This incidence is lower in Scandinavian countries compared to Southern Europe. An increase in the incidence observed in Turkey is associated with the development of bee-keeping in that country $[4,5,6]$.

Polish data originates from the study by Nittner-Marszalska et al. conducted in 2004 [7]; the study revealed a systemic reaction in $8.9 \%$ of the analyzed population (including $4.7 \%$ of I grade reactions in the form of generalized rash), while a large local reaction was noted in $11.8 \%$ of the subjects. The systemic reaction is markedly rarer in children [8]. Furthermore, it is more frequent in males than in females, which is interpreted in terms of the higher exposure of males [4].

Some authors have suggested the probable influence of climatic changes on the incidence of allergic reactions in some areas. An increase in the number of individuals consulting a physician due to sting-related symptoms was observed in Alaska, which can be causally related to an increase in yearly and winter temperature in this region [9].

Analysis of epidemiological data should include a critical approach to the methodology of previous studies. A questionnaire routinely used for survey/history taking represents an imperfect method of analyzing the epidemiology of sensitivity to insect venoms, since it allows 
for a significantly subjective assessment of the episode by the stung individual. The emotional relationship to the event can be vitally important, increasing the risk of misclassifying the type of reaction or the kind of insect that has induced it.

It is possible to perform skin tests and to determine the serum level of specific antibodies against the antigens of insect venoms. Theoretically, these methods could objectively verify the presence of allergy. However, in reality, the presence of sIgE or the positive result of a skin test do not necessarily correspond to allergy; similarly, one cannot exclude the allergy based on the lack of these laboratory indices unless they were tested shortly after the stinging incident. This was confirmed by Nittner-Marszalska et al. [7] among others, who observed positive results of testing (sIgE/skin test) in $53.3 \%$ of individuals with systemic reaction, as well as in $42.8 \%$ of subjects with large local reaction, and in $17.1 \%$ of asymptomatic patients [7].

The incidence of anaphylactic reaction caused by Hymenoptera sting in the US population is estimated at $0.5-5 \%$ [10]. Every year, this type of reaction is responsible for more than 40 deaths in the USA and nearly 100 deaths in Europe [11]. However, these figures can be underestimated as suggested by the results of postmortem analysis of serum samples in cases of unexpected, sudden death due to an unexplained cause. An elevated titer of specific IgE against the antigens of Hymenoptera was detected in $23 \%$ of the cases [12].

Venom. Stings of the various representatives of Hymenoptera differ in terms of the volume of venom injected during each sting. The content of protein per single injection of venom amounts to $52-66 \mu \mathrm{g}$ in the bee, $4.2-17 \mu \mathrm{g}$ in the wasp, and $10-31 \mu \mathrm{g}$ in the bumblebee [13]. While a bee dies immediately after injecting venom, the wasp can sting several times. Additionally, the venoms of these two insects differ in terms of composition [14].

Bee venom contains melittin (membranous toxin), apamin (neurotoxin), tertiapin, MCD-peptide (mast cell degranulating peptide), acidic phosphatase, as well as phospholipase A2 and hyaluronidase, both possessing allergenic properties.

Wasp venom is more immunogenic. The mean number of stings preceding the occurrence of allergic reaction is lower than in the bee, which suggests that the development of allergy to wasp venom does not require such extensive exposure to allergen as in the case of bee venom [15]. In the case of wasp venom, it is important to note mastoparan and kinines, both responsible for its toxic effect, as well as such allergens as phospholipase A and B, hyaluronidase, antigen 5 , acidic phosphatase, and alkaline phosphatase.

An individual can be sensitive to one or more of the aforementioned allergens. Moreover, there might be crossreactivity due to the similarity of epitopes of antigens contained in venoms of various insect species. This may lead to a situation in which an individual becomes sensitized via the sting of an insect belonging to one species, while the allergic reaction is induced by a subsequent sting by the representative of another Hymenoptera species. Bee and bumblebee venoms are characterized by a considerable crossreactivity, while a cross-reaction has not been documented between the allergens of bee and wasp venoms $[2,16]$. Additionally, there is cross-reactivity between the venoms of yellow jacket wasps and hornets [17].
Pathomechanism. Gell and Coombs' type I reaction is acknowledged as being the most common type of immune response in the case of insect allergy. First contact with the allergen present in the venom leads to the synthesis of specific IgE antibodies against this antigen by plasmatocytes (transformed from sensitized B lymphocytes). Subsequent contact with this antigen is reflected by antigen bridging of antibodies bound to the surface of mast cells, which leads to their degranulation, i.e. the release of preformed mediators, among which histamine is the most important. Some of these mediators (prostaglandins, leukotrienes) are synthesized de novo. The release of these molecules causes increased permeability of capillaries, dilatation of venous vessels, and constriction of bronchial and alimentary tract muscular membrane; this is reflected by the cascade of clinical signs leading to the anaphylactic reaction [14].

Mechanisms leading to the activation of specific IgE synthesis are complex. An important role is played by enhanced synthesis of interleukins IL- 4 and IL-13, as well as by the predominance of Th2 phenotype in lymphocyte population. Activation of CD40 on the surface of B lymphocyte by its ligand present on the surface of T lymphocyte (CD40L) is an interaction required for the formation of memory $\mathrm{B}$ cells. This reaction leads to the synthesis of antibodies against T-cell dependent antigens, and inhibits the apoptosis of B lymphocytes [18].

Clinical manifestation. Reactions to Hymenoptera sting can be of both non-allergic and allergic character. Physiological reaction is described as a painful, occasionally itchy wheal, up to $2 \mathrm{~cm}$ in diameter, surrounded by a swelling involving subcutaneous tissue, up to $10 \mathrm{~cm}$ in diameter, persisting for no longer than 24 hours [1].

Toxic reactions result from the cytotoxic effects of specific components of the venom, and are associated with the injection of large amounts of venom to an individual being attacked by a hive of insects. Such an episode can be associated with hemolysis and rhabdomyolysis, with subsequent, potentially lethal renal injury [19].

Allergic reactions are classified as local or systemic. Local allergic reaction pertains to a swelling and rash larger than $10 \mathrm{~cm}$ and persisting for more than 24 hours [1]. This type of reaction is referred to as 'a large local reaction' in Englishspeaking literature; it can be dangerous if located in the region of the upper airways (therefore, bites within the region of the head and neck are potentially most dangerous). However, in reality, the swelling resulting from neck sting is usually markedly smaller than that located within a limb [18].

The clinical manifestation of systemic allergic reaction can be highly variable. The four grade classification system proposed by Müller [1] remains the most popular scheme for classifying systemic reactions:

- Grade I: corresponds to itch, generalized urticaria, weakness, and anxiety;

- Grade II: vasomotor edema, chest tightness, nausea, vomiting, diarrhea, and dizziness;

- Grade III: dyspnea, laryngeal stridor, dysphagia, dysarthria, dysphonia, and fear of death;

- Grade IV: decrease in arterial pressure, collapse, loss of consciousness, urinary and fecal incontinence, and cyanosis.

Usually, the systemic reaction develops within 10 minutes of stinging [18]. 
Significant factors modulating the severity of the reaction include [20, 21]: patient's age, comorbidities, ingested medications (in particular, beta-blockers and angiotensin convertase inhibitors), time gap between consecutive stings and their number, type of reaction observed after a subsequent episode, confirmed sensitivity to venom, baseline level of serum tryptase, and the type of insect. Systemic reaction in children is mild in $60 \%$ of the cases, and the prognosis regarding the manifestation of the reaction after subsequent stinging is favourable.

In contrast, there is a risk of severe systemic reaction in older people due to the frequent presence of comorbidities and medications used to treat these conditions. Asthma and other chronic respiratory conditions with severe manifestation, poorly controlled cardiovascular disorders, and mastocytosis increase the risk of severe reaction. In contrast, this risk decreases with an increase in the time gap between the incidents; nevertheless, remaining at a constant level of $20-30 \%$, even 10 years after the last incident. Conversely, extremely frequent exposure seems to induce tolerance, as suggested by the results of studies in bee-keepers. The history of severe reaction increases the risk of similar systemic reaction in the future. Also, an elevated level of specific IgE constitutes a risk factor of systemic reaction, independently from the history of previous reactions. Irrespective of mastocytosis, which can co-exist in $7-8 \%$ of the cases, an elevated baseline level of tryptase is associated with the occurrence of extremely severe, frequently lethal, systemic reactions $[22,23,24]$.

Experiencing systemic reaction to insect venom is traumatic for the victim and his/her family. It is reflected by emotional stress and decreased health-related quality of life [25].

Diagnosis. Diagnosis of Hymenoptera venom allergy is based on history and positive result of skin tests for revealing the presence of specific IgE in the serum [20]. History remains the most important component of the diagnostic process. Therefore, it should be taken carefully; the patient should be asked what the insect looked like, if the sting was left at the injection site, and what were the circumstances and the place of the incident (whether or not the insect was provoked). These facts are important since the proper selection of immunotherapy requires identifying the culprit. Moreover, the patient should also be asked about previous stinging incidents, type and severity of resultant symptoms, risk factors of systemic reaction and further exposure, any interim treatment, and the time gap between stinging and appearance of the symptoms.

Skin tests include skin prick tests and intradermal tests. It is recommended that these should be performed at least two weeks after the stinging episode as there is a risk of a false negative result due to the refractory period. If the result is negative, but history points to an evident hypersensitivity reaction, the tests should be repeated after 1-2 months due to the possibility of prolonged refractory period. Due to its higher sensitivity, the intradermal test should be performed to confirm a negative result of skin prick testing. Both types of tests involve gradually increasing concentrations of examined substances.

The level of sIgE is determined by RAST method (radioallergosorption test), as well as by its modern, more sensitive modalities (FAST, ImmunoCAP) [26, 27]. The pattern of the increase in the specific IgE during the first few post-stinging days or weeks, and the subsequent decrease after several months, has high individual variability. Therefore, if the result is negative, the test should be repeated after several weeks. New tests are developed with the aim of improve sensitivity and specificity of diagnosis (e.g. IMMULITE) [28].

In about $30-50 \%$ of cases, allergy to insect venoms is associated with the presence of IgE against both bee and wasp venom. This phenomenon is only partially associated with true cross-reactivity; in the great majority of cases this results rather from the reaction with carbohydrate epitopes (CCD - Carbohydrate Cross-reactive Determinants) of allergens, the role of which is not fully understood. Attempts are being made to employ recombinant allergens (lacking CCD), raising the hope for developing methods enabling elimination of this phenomenon in diagnostic tests $[20,29$, 30, 31].

RAST inhibition test is a method used to distinguish between genuine dual sensitivity (being an indication to immunotherapy with vaccines made of both venoms) and cross- reactivity to hyaluronidase or CCD epitopes. The precise diagnosis in these cases, however, is difficult to achieve and requires conducting numerous additional tests which are not routinely made [32]. It has been proved that Vespidae component-specific IgE has value for the diagnosis of venom allergy, as well as for monitoring the effects of venom immunotherapy $[33,34,35]$.

Asymptomatic allergy constitutes an additional significant issue. Elevated serum levels of sIgE are quite frequently detected in individuals with no history of hypersensitivity. However, the specificity of this method with regards to hypersensitivity is still open to discussion. A number of reports indicate that specific antibodies are frequently detected in individuals with an elevated level of total IgE [31].

Provocation challenge, based on controlled stinging by live insect under hospital conditions, is a very efficient diagnostic test used to verify the efficacy of immunotherapy. However, in view of existing controversies (high risk of anaphylactic reaction), the limitation of its application only to scientific purposes is recommended. Other laboratory tests, not routinely used in clinical practice, include basophil activation test, basophil histamine release test, immunoblotting, and leukotriene release test.

All the above-mentioned tests have a low potential with regards to foreseeing the severity of future reactions. Therefore, the diagnostic challenge pertains to developing methods that would enable predicting the consequences of a subsequent stinging episode in the context of a given individual; theoretically, optimizing the selection of candidates for immunotherapy. The poor state of current knowledge on the problem in question is confirmed by the fact that about $50 \%$ of the casualties had no documented history of a previous systemic reaction to Hymenoptera venoms [32]. The natural history of this disease and risk factors of hypersensitivity reaction remain to be elucidated. On the other hand, the risk factors of being re-stung have been specified: proximity of bee-hives and outdoor occupations increase the degree of exposure, and seem to be features of individuals who will benefit most from venom immunotherapy [36].

Prevention and treatment. Patients should be counseled about behaviors that should be avoided in order to reduce the risk of stinging. Sensitive individuals should not provoke 
insects; in particular, they should avoid eating and drinking outside, picking fruits, working in the garden, walking barefoot, staying in the proximity of bee hives during the honey collection seasons, and engaging in outdoor sports [37]. The sting should be removed as soon as possible after being injected as the amount of venom entering the body depends on the time the sting remained in the skin [38].

Anaphylaxis is a type of life-threatening systemic reaction which requires prompt diagnosis and immediate implementation of the treatment. Intramuscular injection of $1 \mathrm{mg} / 1 \mathrm{~mL}$ adrenaline solution $(0.3-0.5 \mathrm{mg}$ in adults or $0.01 \mathrm{mg} / \mathrm{kg}$ in children) is the procedure of choice in such cases. If necessary, the dose can be repeated every 5-15 minutes. An immediate and properly administered injection is vitally important, because it is the only way to guarantee the effectiveness of this procedure. Adrenaline given at the abovementioned dose is not effective if administered at the stage of cardiac arrest $[21,37]$. In the case of anaphylaxis induced by insect venoms, mean time to cardiorespiratory arrest is equal to 15 minutes [39]. Furthermore, a delay in injecting adrenaline increases the probability of biphasic anaphylaxis, i.e. the recurrence of symptoms 1-72 hours (most commonly 8 hours) after resolution of the first phase, despite the absence of a trigger [21]. Patients with cardiovascular comorbidities are at a higher risk of experiencing side-effects associated with the administration of adrenaline. Although the balance of benefits and risks substantiates using this drug in this group of patients, maximal care is advised [21,37].

Other administered drugs include antihistamines acting thorough $\mathrm{H} 1$ receptor (alleviate rash and itching not relieved by adrenaline), beta-2-mimetics (attenuate residual bronchial spasm, which did not respond to adrenaline), and glucocorticoids (prevent biphasic and prolonged anaphylaxis) [21].

For those individuals who have experienced a systemic reaction, it is important to prescribe and explain the proper usage of an intramuscular adrenaline injection kit. It was revealed that only about one-third of patients receive a prescription for an adrenaline kit, and s referred to an allergology specialist for further diagnostics and discussion about potential immunotherapy [39]. Consequently, extensive education is necessary of both the patients and physicians with regards to these issues. Healthcare professionals should be trained to become proficient in the auto-injection kit in order to further educate their patients until perfection in obtained. The patient has to learn how to use the kit with maximal proficiency in order not to make a mistake in a real life-threatening situation, when his/her actions are additionally complicated by high emotions.

Specific immunotherapy with vaccine containing the allergen of insect venom has an established efficacy in reducing the risk of anaphylactic reaction in sensitive individuals [37, 40]. Mechanisms underlying this therapy are complex and mostly not understood. Immunotherapy is reflected by an increased activity of CD4+ CD25+ FOXP3+ subpopulation of regulatory $\mathrm{T}$ lymphocytes, as well as type 1 regulatory $\mathrm{T}$ lymphocytes releasing IL-10. These reactions are seen as beneficial in the context of developing tolerance to the allergen. Additionally, immunotherapy is associated with inhibited activity of eosinophils, mast cells, and basophils, and an elevated level of IgG4 [41].

Immunotherapy with vaccine based on insect venom is recommended in children and adults with a history of severe systemic reaction with circulatory and respiratory symptoms, and who tested positive with regards to skin tests and/or serum sIgE. Potential indications in the case of non-life-threatening systemic reactions (generalized erythematous rash and itching) depend on the presence of risk factors (exposure to stings, circulatory comorbidities, and mastocytosis) or a significant influence of the allergy on the quality of life [37].

Contraindications are similar to those in cases of other types of desensitization. Although pregnancy constitutes a contraindication to the implementation of desensitization, it does not preclude continuation of already started immunotherapy. In patients given beta-adrenolytics, the decision on further treatment (cessation of immunotherapy, discontinuation of beta-blockers, and starting desensitization, or exceptionally, immunotherapy without discontinuation of these agents, completed with comprehensive monitoring of the circulatory system) should be based on analysis of cardiovascular risk and the risk of anaphylaxis [37].

The vaccine is selected based on the identification of the insect (anamnesis, adjunct tests) and based on the knowledge of cross-reactivity between allergens of various venoms [37].

Immunotherapy with the vaccine based on the allergens of the venom is associated with a risk of adverse effects, among which systemic reaction is the most severe. Various attempts are being undertaken in order to reduce the risk of side-effects, e.g. the administration of antihistaminic agents [42], antibodies isolated from bee-keepers' sera [43], or trials using recombinant allergens [44].

Although $100 \mu \mathrm{g}$ is the most commonly used booster dose, some patients require a higher dose for complete protection [45]. Typically, there is a 4-8-week interval between consecutive booster doses, and the therapy lasts for $3-5$ years $[45,46]$. Such a model of immunotherapy provides long-term tolerance in $85 \%$ of patients [46]. However, with time, the patient gradually loses the motivation for frequent visits to the doctor's surgery. The results of recent studies suggest that extending the intervals between booster doses to three months does not negatively influence the treatment efficacy and safety; however, it does increase the probability of completing the scheduled treatment protocol [47]. A study which utilized a provocation challenge consisting of stinging induced by a live bee, revealed that tolerance can be observed immediately after reaching the booster dose. The challenge performed within one week of reaching the booster dose of $100 \mu \mathrm{g}$ was reflected by the lack of any abnormal reaction in $88.6 \%$ of the participants [48].

Aside from the conventional method, the rush or ultra-rush methods may be applied in cases requiring rapid development of tolerance. They are equally effective and safe. The use of anti-histaminic agents in combination with these methods is recommended, because not only do they increase the safety, but they also improve the long-term therapeutic effects of immunotherapy $[42,45]$. However, the health-related quality of life is lower in patients undergoing rush and ultra-rush protocol of immunotherapy, compared to those undergoing conventional therapy [49]. This fact should also be considered when qualifying the patient.

At present, oral immunotherapy of Hymenoptera venom allergy is being investigated; however, the currently available results do not substantiate wide use of this method [50,51].

The effectiveness of venom immunotherapy and its impact on the quality of life has been proved in a high- 
quality systematic review [52]. Moreover, it seems to be costeffective, but it is important to have those patients under strict observation to select groups of patients who benefit most from this form of therapy [53].

\section{CONCLUSION}

Currently conducted studies are aimed at a better understanding of the natural history of Hymenoptera venom allergy and its pathomechanisms in order to improve diagnostic and therapeutic methods. The development of diagnostic methods able to predict the severity of the reaction to future stinging episodes seems particularly important. At present, extensive studies on recombinant antigens are being performed in order to improve both diagnostic and therapeutic methods [54]. Irrespective of the development of new research targets, using the already possessed knowledge is also important. The training of healthcare professionals should be optimized to provide the proper management of affected patients. The levels of knowledge and awareness with regards to the effectiveness of immunotherapy are low, both in society and amongst healthcare personnel [55]. Although Hymenoptera venom allergy is relatively rare, it can be potentially life threatening; therefore, it is important to provide specialist care for every patient seeking medical attention due to an episode of Hymenoptera venom hypersensitivity.

\section{REFERENCES}

1. Müller UR. Entomology of Hymenoptera. Clinical presentation and pathogenesis. In: Muller UR Insect Sting allergy: clinical picture, diagnosis and treatment. Gustav Fisher, New York 1990: 3-65.

2. de Groot H. Allergy to bumblebees. Curr Opin Allergy Clin Immunol. 2006; 6: 294-7.

3. Tankersley MS. The stinging impact of the imported fire ants. Curr Opin Allergy ClinImmunol. 2008; 8: 354-9.

4. Björnsson E, Janson C, Plaschke P, Norrman E, Sjöberg O. Venom allergy in adult Swedes: a population study. Allergy.1995; 50: 800-5.

5. Kalyoncu AF, Demir AU, Ozcan U, Ozkuyumcu C, Sahin AA, Baris YI. Bee and wasp venom allergy in Turkey. Ann Allergy Asthma Immunol. 1997; 78: 408-12.

6. Golden DB, Marsh DG, Kagey-Sobotka A, et al. Epidemiology of insect venom sensitivity. JAMA. 1989; 262(2): 240-4

7. Nittner-Marszalska M, Liebhart J, Liebhart E, et al. Prevalence of Hymenoptera venom allergy and its immunological markers current in adults in Poland. Med Sci Monit. 2004; 10: 324-9.

8. Novembre E, Cianferoni A, Bernardini R, et al. Epidemiology of insect venom sensitivity in children and its correlation to clinical and atopic features. Clin Exp Allergy. 1998; 28: 834-8.

9. Demain JG, Gessner BD, McLaughlin JB, Sikes DS, Foote JT. Increasing insect reactions in Alaska: is this related to changing climate? Allergy Asthma Proc. 2009; 30: 238-43.

10. Neugut AI, Ghatak AT, Miller RL. Anaphylaxis in the United States: an investigation into its epidemiology. Arch Intern Med. 2001; 161: 15-21.

11. Müller UR. Hymenoptera venom hypersensitivity: an update. Clin Exp Allergy. 1998; 28: 4-6.

12. Schwartz HJ, Sutheimer C, Gauerke MB, Yunginger JW. Hymenoptera venom-specific IgE antibodies in post-mortem sera from victims of sudden, unexpected death. Clin Allergy. 1988; 18: 461-8.

13. Hoffman DR, Jacobson RS. Allergens in hymenoptera venom XII: how much protein is in a sting? Ann Allergy. 1984; 52: 276-8.

14. Szumera B, Lalik B, Szlęk R. Współczesne osiągnięcia w dziedzinie alergii na jad owadów błonkoskrzydłych [Present achievements in treatment of allergy to the hymenopteral insects venom]. Przegl Pediatr. 2005; 35: 137-143 (in Polish).

15. Ewan PW. Allergy to insect stings: a review. J R Soc Med. 1985; 78: 234-9.
16. Reisman RE, Müller UR, Wypych JI, Lazell MI. Studies of coexisting honeybee and vespid-venom sensitivity. J Allergy ClinImmunol. 1984; 73: 246-52.

17. Mosbech H. Anaphylaxis to insect venom. Novartis Found Symp. 2004; 257: 177-88.

18. Annila I. Bee venom allergy. Clin Exp Allergy. 2000; 30: 1682-7.

19. Vetter RS, Visscher PK, Camazine S. Mass envenomations by honey bees and wasps. West J Med. 1999; 170: 223-7.

20. Biló BM, Rueff F, Mosbech H, et al. EAACI Interest Group on Insect Venom Hypersensitivity: Diagnosis of Hymenoptera venom allergy. Allergy 2005; 60: 1339-49.

21. Simons FE. Anaphylaxis. J Allergy Clin Immunol. 2010; 125: 161-81.

22. Marciniak J, Jutel M. Znaczenie mastocytozy w reakcjach anafilaktycznych. Post Dermatol Alergol. 2006; XXIII, 1: 17-20.

23. Ludolph-Hauser D, Ruëff F, Fries C, Schöpf P, Przybilla B. Constitutively raised serum concentrations of mast-cell tryptase and severe anaphylactic reactions to Hymenoptera stings. Lancet. 2001; 357: 361-362.

24. Haeberli G, Bronnimann M, Hunziker T, Muller U. Elevated basal serum tryptase and Hymenoptera venom allergy: relation to severity of sting reactions and to safety and efficacy of venom immunotherapy. Clin Exp Allergy. 2003; 33: 1216-1220.

25. Oude-Elberink JNG, de Monchy JGR, Goldek DBK, et al. Development and validation of health-related quality of life questionnaire in patients with yellow jacket allergy. J Allergy Clin Immunol. 2002; 109: 162-170.

26. Jeep S, Kircholf E, O’Connor A, Kunkel G. Comparison of Phadebas RAST with the Pharmacia CAP system for insect venom. Allergy. 1992; 47: 212-217.

27. Alonso R, Botey J, Pena JM, Eseverri JL, Marin A, Ras RM. Specific IgE determination using the CAP system: comparative evaluation with RAST. J Investig Allergol ClinImmunol. 1995; 5: 156-60.

28. Watanabe M, Hirata H, Arima M, et al. Measurement of Hymenoptera venom specific IgE by the IMMULITE3 3gAllergy in subjects with negative or positive results by ImmunoCAP. Asia Pac Allergy. 2012; 2:195-202.

29. Seismann H, Blank S, Braren I, et al. Dissecting cross-reactivity in hymenoptera venom allergy by circumvention of alpha-1,3-core fucosylation. Mol Immunol. 2010; 47: 799-808.

30 . Hemmer W. Cross-reactivity to honeybee and wasp venom. Hautarzt. 2008; 59: 194-9.

31. Erzen R, Korosec P, Silar M, Music E, Kosnik E. Carbohydrate epitopes as a cause of cross-reactivity in patients allergic to Hymenoptera venom. Wien Klin Wochenschr. 2009; 121: 349-52.

32. Neis MM, Merk HF. Value of component based diagnostics in IgEmediated hymenoptera sting reactions. Cutan Ocul Toxicol. 2012; 31: $117-123$.

33. Shin YS, Liu JN, Hur GY, et al. Clinical Features and the Diagnostic Value of Component Allergen-Specific IgE in Hymenoptera Venom Allergy. Allergy Asthma Immunol Res. 2012; 4: 284-289.

34. Sturm GJ, Schuster C, Kranzelbinder B, Wiednig M, Groselj-Strele A, Aberer W. Asymptomatic sensitization to hymenoptera venom is related to total immunoglobulin E levels. Int Arch Allergy Immunol. 2009; 148: 261-4.

35. Bilò MB, Bonifazi F. The natural history and epidemiology of insect venom allergy: clinical implications. Clin Exp Allergy. 2009; 39: 146776

36. von Moos S, Graf N, Johansen P, Muller G, Kundig TM, Senti G. Risk assessment of Hymenoptera re-sting frequency: implications for decision-making in venomimmunotherapy. Int Arch Allergy Immunol. 2013; 160: 86-92.

37. Bonifazi F, Jutel M, Biló BM, et al. EAACI Interest Group on Insect Venom Hypersensitivity. Prevention and treatment of hymenoptera venom allergy: guidelines for clinical practice. Allergy. 2005; 60: 145970

38. Visscher PK, Vetter RS, Camazine S. Removing bee stings. Lancet. 1996; 348: 301-302.

39. Pumphrey RS. Lessons for management of anaphylaxis from a study of fatal reactions. Clin Exp Allergy. 2000; 30: 1144-50.

40. Münstedt K, Wrobel D, Kalder M. Efficacy of venom immunotherapy in beekeepers. J Investig Allergol Clin Immunol. 2010; 20: 58-62.

41. Jutel M, Akdis CA. Immunological mechanisms of allergen-specific immunotherapy. Allergy. 2011; 66: 725-732.

42. Scribner TA, Bernstein DI. Rapid venom immunotherapy update. Curr Opin Allergy Clin Immunol. 2003; 3: 295-8.

43. Przybilla B, Ring J, Galosi A, Geursen RG, Stickl HA. Bee venom immunoglobulin for prophylaxis of anaphylactic reactions during bee venom immunotherapy (rush hyposensitization). Immunol Allergy Pract. 1986; 8: 107-111. 
44. Müller UR. Recombinant Hymenoptera venom allergens. Allergy. 2002; 57: $570-576$.

45. Golden DB. Insect sting allergy and venom immunotherapy: a model and a mystery. J Allergy Clin Immunol. 2005; 115: 439-47.

46. Golden DB. Insect sting allergy and venom immunotherapy. Ann Allergy Asthma Immunol. 2006; 96: 16-21.

47. Cavallucci E, Ramondo S, Renzetti A, et al. Maintenance venom immunotherapy administered at a 3-month interval preserves safety and efficacy and improves adherence. J Investig Allergol Clin Immunol. 2010; 20: 63-8.

48. Goldberg A, Confino-Cohen R. Bee venom immunotherapy - how early is it effective? Allergy. 2010; 65: 391-395.

49. Cichocka-Jarosz E, Brzyski P, Świebocka E, et al. Health-related quality of life in Polish adolescents with Hymenoptera venom allergy treated with venom immunotherapy. Arch Med Sci. 2012; 8:1076-1082.

50. Severino MG, Cortellini G, Bonadonna P, et al. Sublingual immunotherapy for large local reactions caused by honeybee sting: a double-blind, placebo-controlled trial. J Allergy Clin Immunol. 2008; 122: 44-48.
51. Ruëff F, Bilò MB, Jutel M, et al. Interest group on Hymenoptera venom allergy of the European Academy of Allergology and Clinical Immunology. Sublingual immunotherapy with venom is not recommended for patients with Hymenoptera venom allergy. J Allergy Clin Immunol. 2009; 123: 272-3.

52. Boyle RJ, Elremeli M, Hockenhull J, et al. Venom immunotherapy for preventing allergic reactions to insect stings. Cochrane Database Syst Rev. 2012; 10; CD008838. DOI: 10.1002/14651858.CD008838.pub2.

53. Hockenhull J, Elremeli M, Cherry MG, et al. A systematic review of the clinical effectiveness and cost-effectiveness of Pharmalgen ${ }^{\star}$ for the treatment of bee and wasp venom allergy. Health Technol Assess. 2012; 16(12).

54. Seismann H, Blank S, Cifuentes L, et al. Recombinant phospholipase A1 (Ves v 1) from yellow jacket venom for improved diagnosis of hymenoptera venom hypersensitivity. Clin Mol Allergy. 2010; 8: 7.

55. Bilò BM, Bonifazi F. Epidemiology of insect-venom anaphylaxis. Curr Opin Allergy Clin Immunol. 2008; 8: 330-7. 